\title{
Adaptive Iterated Extended Kalman Filter and Its Application to Autonomous Integrated Navigation for Indoor Robot
}

\author{
Yuan $\mathrm{Xu},{ }^{1,2}$ Xiyuan Chen, ${ }^{1,2}$ and Qinghua $\mathrm{Li}^{1,3}$ \\ ${ }^{1}$ School of Instrument Science and Engineering, Southeast University, Nanjing, China \\ ${ }^{2}$ Key Laboratory of Micro-Inertial Instrument and Advanced Navigation Technology, Ministry of Education, Nanjing, China \\ ${ }^{3}$ School of Electrical Engineering and Automation, Qilu University of Technology, Jinan, China \\ Correspondence should be addressed to Xiyuan Chen; chxiyuan@seu.edu.cn
}

Received 24 October 2013; Accepted 30 December 2013; Published 13 February 2014

Academic Editors: S. Balochian, V. Bhatnagar, and Y. Zhang

Copyright (c) 2014 Yuan Xu et al. This is an open access article distributed under the Creative Commons Attribution License, which permits unrestricted use, distribution, and reproduction in any medium, provided the original work is properly cited.

\begin{abstract}
As the core of the integrated navigation system, the data fusion algorithm should be designed seriously. In order to improve the accuracy of data fusion, this work proposed an adaptive iterated extended Kalman (AIEKF) which used the noise statistics estimator in the iterated extended Kalman (IEKF), and then AIEKF is used to deal with the nonlinear problem in the inertial navigation systems (INS)/wireless sensors networks (WSNs)-integrated navigation system. Practical test has been done to evaluate the performance of the proposed method. The results show that the proposed method is effective to reduce the mean root-meansquare error (RMSE) of position by about $92.53 \%, 67.93 \%, 55.97 \%$, and $30.09 \%$ compared with the INS only, WSN, EKF, and IEKF.
\end{abstract}

\section{Introduction}

As the development of automation indoor mobile robots, how to obtain accurate navigation information of indoor mobile robots has received great attention over the past few decades.

To the integrated system, the global positioning systems (GPS)/inertial navigation systems (INS) integrated system is one of the most used methods for the outdoor navigation. Many attempts try to improve the accuracy of the GPS/INS integration. For example, Quinchia et al. compared different error modeling of MEMS applied to GPS/INS integrated systems in [1], Jwo et al. proposed a fuzzy adaptive strong tracking unscented Kalman filter for ultratight GPS/INS integrated systems [2], Chen et al. proposed a GPS/INS system using novel filtering methods for vessel attitude determination [3], and Jwo et al. proposed a nonlinear filtering with IMM algorithm for ultratight GPS/INS integration [4]. Meanwhile, in order to overcome the GPS outage, some attempts try to design bridge methods by using the artificial intelligence algorithms [5] such as Neural Networks (NN) [6-8] and least squares support vector machine (LS-SVM) [9-11]. However, since the accuracy of the integrated system is depending on the GPS, it has poor performance in the indoor environment.
In order to get higher positioning accuracy indoor, some literatures try to employ wireless localization to replace the GPS in the integrated system. For instance, S. J. Kim and B. K. Kim proposed an accurate hybrid global self-localization algorithm for indoor mobile robots with two-dimensional isotropic ultrasonic receivers [12], and an accurate pedestrian indoor navigation by tightly coupling foot-mounted IMU and RFID measurements was proposed in [13]. On the basis of the navigation strategy, the data fusion algorithm should also be designed seriously. In this field, Kalman filter (KF) is able to achieve the optimal state estimation [14]. However, it is not suitable for nonlinear systems. Thus, the extended KF (EKF) is proposed to overcome this problem by Taylor series expansion, which may introduce a truncated error [15]. In order to overcome this problem, the iterated EKF (IEKF) is proposed. However, the data fusion algorithms mentioned above are difficult to track the accurate state during the target's fast movement since it employs a fixed priori estimates for the process and measurement noise covariances during the whole estimation process [16].

In order to overcome these problems, we employed the noise statistics estimator in the IEKF, which combines the advantages of the AEKF and the IEKF. The remainder of 
the paper is organized as follows. Sections 2 and 3 give the introduction for AIEKF and its application to INS/WSN integrated system. The tests and discussion are illustrated in Section 4. Finally, the conclusions are given.

\section{Adaptive Iterated Extended Kalman Filter}

In this section, a brief introduction to the EKF and IEKF will be given, and then AIEKF will be proposed. It is assumed that a discrete-time model of a nonlinear system is given by

$$
\begin{aligned}
& \mathbf{x}_{k}=f\left(\mathbf{x}_{k-1}\right)+\omega_{k}, \\
& \mathbf{y}_{k}=h\left(\mathbf{x}_{k}\right)+v_{k},
\end{aligned}
$$

where $\mathbf{x}_{k}$ and $\mathbf{y}_{k}$ are the state vector and the measurement vector for the filter, $f(\cdot)$ and $h(\cdot)$ are the dynamic model function and the measurement function, respectively, and $\omega_{k}$ and $v_{k}$ are the process noise vector and measurement noise vector, respectively. It is assumed that $\omega_{k}$ and $v_{k}$ are independent zero-mean white Gaussian sequences with covariance $\mathbf{Q}$ and $\mathbf{R}$, respectively.

2.1. Extended Kalman Filter. The traditional EKF algorithm is utilizing a set of equations as follows [17]:

$$
\begin{gathered}
\widehat{\mathbf{X}}_{k \mid k-1}=\mathbf{A}_{k \mid k-1} \widehat{\mathbf{X}}_{k-1 \mid k-1}+\widehat{\mathbf{q}}_{k}, \\
\mathbf{P}_{k \mid k-1}=\mathbf{A}_{k \mid k-1} \mathbf{P}_{k-1} \mathbf{A}_{k \mid k-1}^{T}+\widehat{\mathbf{Q}}_{k}, \\
\mathbf{K}_{k}=\mathbf{P}_{k \mid k-1} \mathbf{H}_{k}^{T}\left[\mathbf{H}_{k} \mathbf{P}_{k \mid k-1} \mathbf{H}_{k}^{T}+\widehat{\mathbf{R}}_{k}\right]^{-1}, \\
v_{k}=\mathbf{y}_{k}-\mathbf{h}\left(\widehat{\mathbf{X}}_{k \mid k-1}\right), \\
\widehat{\mathbf{X}}_{k \mid k}=\widehat{\mathbf{X}}_{k \mid k-1}+\mathbf{K}_{k} v_{k}, \\
\mathbf{P}_{k \mid k}=\left[\mathbf{I}-\mathbf{K}_{k} \mathbf{H}\left(\widehat{\mathbf{X}}_{k \mid k}\right)\right] \mathbf{P}_{k \mid k-1},
\end{gathered}
$$

where $\mathbf{A}_{k \mid k-1}=\partial \mathbf{f}\left(\widehat{\mathbf{X}}_{k \mid k}\right) / \partial \widehat{\mathbf{X}}_{k \mid k}, \mathbf{H}_{k}=\partial \mathbf{h}\left(\widehat{\mathbf{X}}_{k \mid k}\right) / \partial \widehat{\mathbf{X}}_{k \mid k}$.

2.2. Iterated Extended Kalman Filter. Compared with the EKF, the IEKF employs a few simple iterative operations to reduce the bias and the estimation error after getting $\mathbf{X}_{k}$ in (2) and $\mathbf{P}_{k}$ in (3). The corresponding recursive relations are

$$
\begin{gathered}
\widehat{\mathbf{X}}_{k \mid k}^{(1)}=\widehat{\mathbf{X}}_{k \mid k-1}, \\
\mathbf{P}_{k \mid k}^{(1)}=\mathbf{P}_{k \mid k-1}, \\
\mathbf{K}_{k}^{(n)}=\mathbf{P}_{k \mid k-1}\left(\mathbf{H}^{(n)}\right)^{T}\left[\mathbf{H}^{(n)} \mathbf{P}_{k \mid k-1}\left(\mathbf{H}^{(n)}\right)^{T}+\mathbf{R}\right]^{-1}, \\
\widehat{\mathbf{X}}_{k \mid k}^{(n+1)}=\widehat{\mathbf{X}}_{k \mid k}^{(n)}+\mathbf{K}_{k}^{(n)}\left[\mathbf{y}_{k}-\mathbf{h}^{(n)}\left(\widehat{\mathbf{X}}_{k \mid k}^{(n)}\right)-\mathbf{H}^{(n)}\right. \\
\left.\times\left(\widehat{\mathbf{X}}_{k \mid k-1}-\widehat{\mathbf{X}}_{k \mid k}^{(n)}\right)\right], \\
\mathbf{P}_{k \mid k}^{(n)}=\left[\mathbf{I}-\mathbf{K}_{k}^{(n)} \mathbf{H}^{(n)}\right] \mathbf{P}_{k \mid k-1}^{(n)},
\end{gathered}
$$

where $\mathbf{H}^{(n)}=\partial \mathbf{h}\left(\widehat{\mathbf{X}}_{k \mid k}^{n}\right) / \partial \widehat{\mathbf{X}}_{k \mid k}^{n}$ and the superscript $n(n=1$, $2, \ldots, m)$ is the number of iteration steps, And then,

$$
\begin{aligned}
\widehat{\mathbf{X}}_{k \mid k} & =\widehat{\mathbf{X}}_{k \mid k}^{(m)}, \\
\mathbf{P}_{k \mid k} & =\mathbf{P}_{k \mid k}^{(m)} .
\end{aligned}
$$

2.3. Adaptive Iterated Extended Kalman Filter. The EKF overcomes the nonlinear problem by ignoring the higher order terms, which may introduce a truncated error. Thus, the IEKF overcomes this problem. However, it is evident that both the $\mathbf{Q}$ and $\mathbf{R}$ for EKF and those for IEKF are prior estimates. In practice, there are uncertainties in the noise description, and the assumptions on the statistics of disturbances are violated since the availability of precisely known model is unrealistic practical situations. In order to overcome these problems, we employed the noise statistics estimator into the IEKF. Meanwhile, when the system noise is stable and the error variance is small, it is able to employ observation noise estimator only. The corresponding recursive relations are

$$
\begin{gathered}
\widehat{\mathbf{X}}_{k \mid k}^{(1)}=\widehat{\mathbf{X}}_{k \mid k-1}, \\
\mathbf{P}_{k \mid k}^{(1)}=\mathbf{P}_{k \mid k-1}, \\
\mathbf{K}_{k}^{(n)}=\mathbf{P}_{k \mid k-1}\left(\mathbf{H}^{(n)}\right)^{T}\left[\mathbf{H}^{(n)} \mathbf{P}_{k \mid k-1}\left(\mathbf{H}^{(n)}\right)^{T}+\widehat{\mathbf{R}}_{k-1}^{(n)}\right]^{-1} \\
\widehat{\mathbf{X}}_{k \mid k}^{(n+1)}=\widehat{\mathbf{X}}_{k \mid k}^{(n)}+\mathbf{K}_{k}^{(n)}\left[\mathbf{y}_{k}-\mathbf{h}^{(n)}\left(\widehat{\mathbf{X}}_{k \mid k}^{(n)}\right)-\mathbf{H}^{(n)}\right. \\
\left.\times\left(\widehat{\mathbf{X}}_{k \mid k-1}-\widehat{\mathbf{X}}_{k \mid k}^{(n)}\right)\right] \\
\mathbf{P}_{k \mid k}^{(n)}=\left[\mathbf{I}-\mathbf{K}_{k}^{(n)} \mathbf{H}^{(n)}\right] \mathbf{P}_{k \mid k-1}^{(n)},
\end{gathered}
$$

where $\widehat{\mathbf{R}}_{k}^{(n)}$ is computed by the time-varying noise statistics estimators with the following equations:

$$
\begin{aligned}
\widehat{\mathbf{R}}_{k}^{(n)}= & \left(1-d_{k-1}\right) \widehat{\mathbf{R}}_{k-1}^{(n)} \\
& +d_{k-1}\left(\left[\mathbf{I}-\mathbf{H}_{k}^{(n)} \mathbf{K}_{k}\right] v_{k} v_{k}^{T}\left[\mathbf{I}-\mathbf{H}_{k}^{(n)} \mathbf{K}_{k}\right]^{T}\right. \\
& \left.+\mathbf{H}_{k}^{(n)} \mathbf{P}_{k \mid k-1}^{(n)}\left(\mathbf{H}_{k}^{(n)}\right)^{T}\right),
\end{aligned}
$$

here, $d_{k-1}=(1-b) /\left(1-b^{k}\right), 0<b<1$. And then,

$$
\begin{aligned}
\widehat{\mathbf{X}}_{k \mid k} & =\widehat{\mathbf{X}}_{k \mid k}^{(m)}, \\
\mathbf{P}_{k \mid k} & =\mathbf{P}_{k \mid k}^{(m)}, \\
\mathbf{R}_{k} & =\mathbf{R}_{k}^{(m)}
\end{aligned}
$$

\section{Adaptive Iterated Extended Kalman Filter for Integrated Navigation}

In this work, we just consider the navigation information for mobile robot in the relative coordinate. The INS error is the accumulation of errors in each time. In order to achieve 


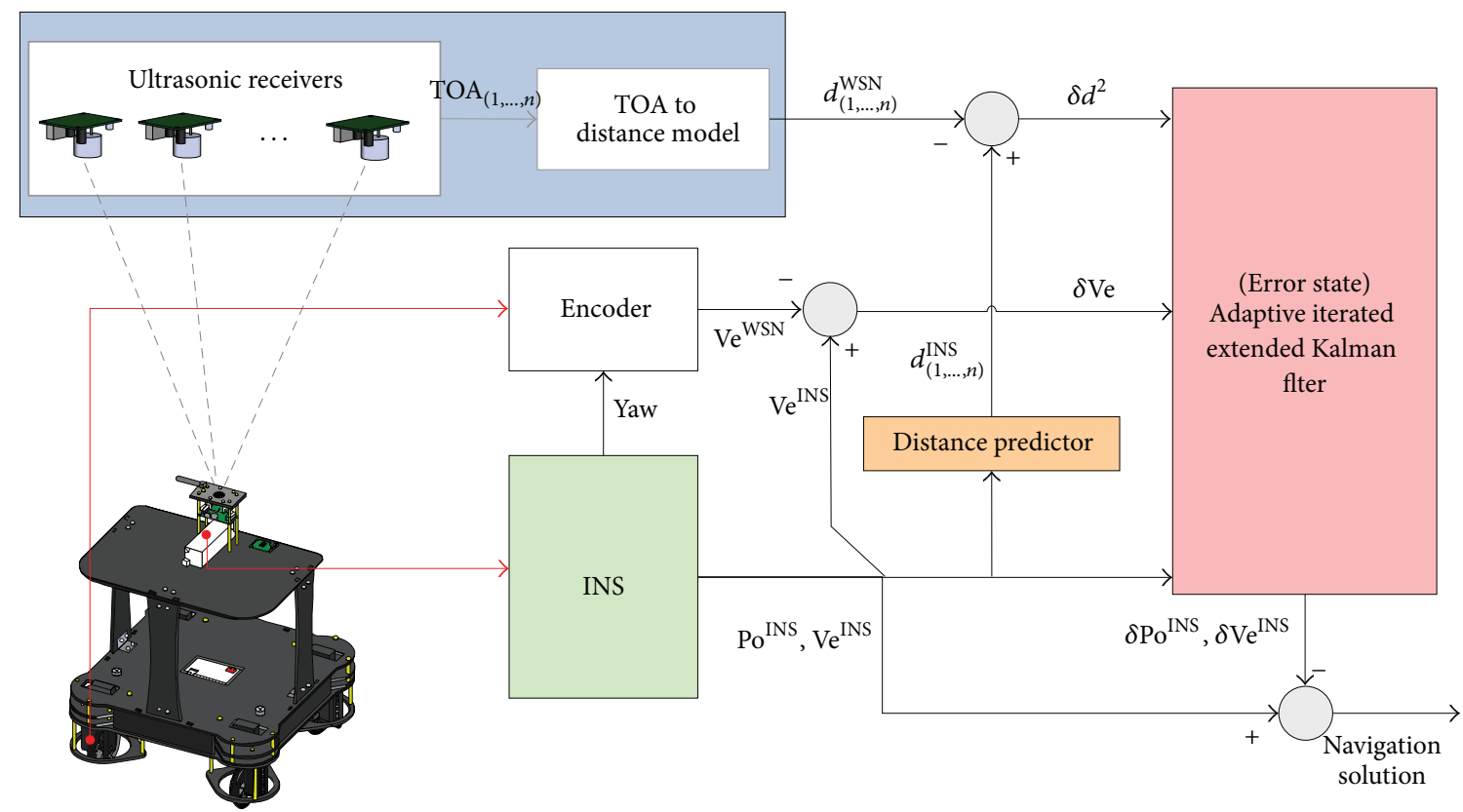

FIGURE 1: Configuration of the hybrid system.

better estimation accuracy of INS error, the state vector is defined by $\mathbf{x}=\left[\delta P_{E} \delta P_{N} \delta V_{E} \delta V_{N} \delta \mathrm{Acc}_{E} \delta \mathrm{Acc}_{N}\right]$. Here, $\left(\delta P_{E, k}, \delta P_{N, k}\right),\left(\delta V_{E, k}, \delta V_{N, k}\right)$, and $\left(\delta A_{C c}, k=A c c_{N, k}\right)$ are the errors of position, velocity, and accelerometer measured by INS in east and north direction. The system equation for the filter at time $k$ is illustrated in.

$$
\underbrace{\left[\begin{array}{c}
\delta P_{E, k} \\
\delta V_{E, k} \\
\delta \mathrm{Acc}_{E, k} \\
\delta P_{N, k} \\
\delta V_{N, k} \\
\delta \mathrm{Acc}_{N, k}
\end{array}\right]}_{\mathbf{x}_{k}}=\underbrace{\left[\begin{array}{cccccc}
1 & T & T^{2} / 2 & 0 & 0 & 0 \\
0 & 1 & T & 0 & 0 & 0 \\
0 & 0 & 1 & 0 & 0 & 0 \\
0 & 0 & 0 & 1 & T & T^{2} / 2 \\
0 & 0 & 0 & 0 & 1 & T \\
0 & 0 & 0 & 0 & 0 & 1
\end{array}\right]}_{\mathbf{A}} \underbrace{\left[\begin{array}{c}
\delta P_{E, k-1} \\
\delta V_{E, k-1} \\
\delta \mathrm{Acc}_{E, k-1} \\
\delta P_{N, k-1} \\
\delta V_{N, k-1} \\
\delta \mathrm{Ac}_{N, k-1}
\end{array}\right]}_{\mathbf{x}_{k-1}}+\mathbf{W}_{k}
$$

where $T$ is sample time and $\mathbf{W}_{k}$ is the process noise vector. The measurement equation for the filter at time $k$ is illustrated in.

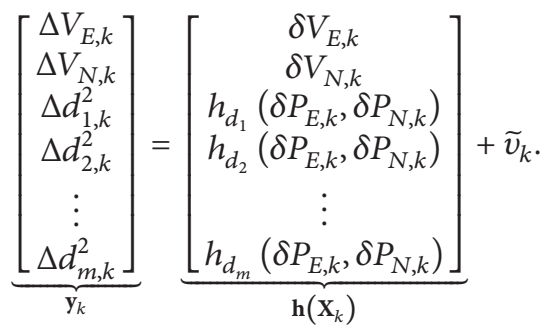

Here, $\Delta d_{i}^{2}$ is the difference between the distance form reference node $(\mathrm{RN})$ to the mobile robot measured by the
INS and WSN, respectively, at time $k$, and it is expressed as follows:

$$
\begin{aligned}
\Delta d_{i, k}^{2}= & \left(d_{i}^{\mathrm{INS}}\right)^{2}-\left(d_{i}^{\mathrm{WSN}}\right)^{2} \\
= & 2\left(P_{E}^{\mathrm{INS}}-P_{E}^{\mathrm{RN}, i}\right) \delta P_{E, k}+2\left(P_{N}^{\mathrm{INS}}-P_{N}^{\mathrm{RN}, i}\right) \delta P_{N, k} \\
& -\left(\delta P_{E, k}^{2}+\delta P_{N, k}^{2}\right), \quad i=1,2, \ldots, m,
\end{aligned}
$$

where $d_{i}^{\mathrm{INS}}$ and $d_{i}^{\mathrm{WSN}}$ are the distances from mobile robot to $i$ th $\mathrm{RN}$ measured by the INS and WSN, respectively, $\left(P_{E}^{\mathrm{INS}}, P_{N}^{\mathrm{INS}}\right)$ is INS position for mobile robot, and $\left(P_{E}^{\mathrm{RN}, i}\right.$, $\left.P_{N}^{\mathrm{RN}, i}\right)$ is $i$ th $\mathrm{RN}$ position. And $\left(\Delta V_{E}, \Delta V_{N}\right)$ is the difference of the WSN and INS velocities in east and north direction, respectively, and $\widetilde{v}_{k}$ is measurement noise vector. The derivation of (15) has a very detailed description in [18]. The configuration of the hybrid system is shown in Figure 1.

\section{Indoor Localization Tests and Discussion}

4.1. The Architecture of the Integrated Navigation System. In this work, a real testbed is built to evaluate the performance of the proposed method. Figure 2 displays the architecture of the testbed. In this work, the mobile robot (shown in Figure 3) is used to run along the preset trajectory. The IMU fixed to the robot are used to provide INS solution for the position, velocity, and the attitude of the mobile robot. The mobile robot position measured by the WSN is used as ultrasonic sender and the receiver. And the computer is used for saving sensor data.

The sample time used in the test is $0.02 \mathrm{~s}$, and the mobile robot runs along the trajectories shown in Figure 4 with $0.3 \mathrm{~m} / \mathrm{s}$. Meanwhile, the RNs are denoted by yellow circles in Figure 4 . 


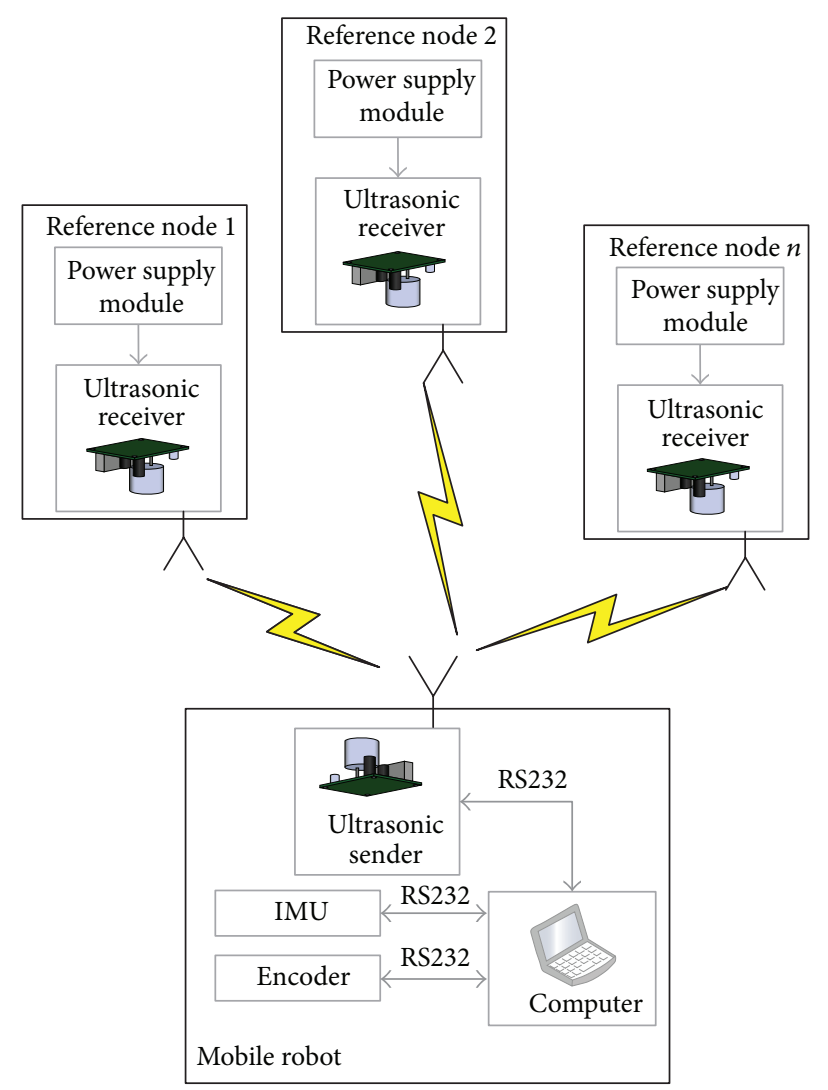

FIGURE 2: The architecture of the integrated navigation system.

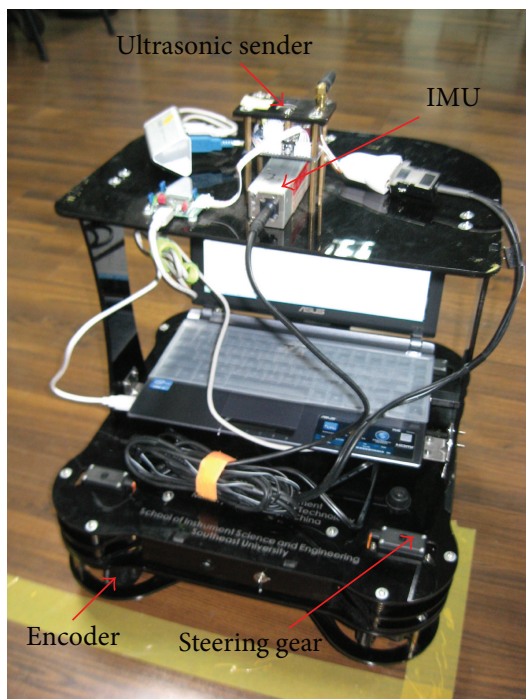

FIgURE 3: The prototype of the robot.

4.2. The Performance of the Proposed Method. In this section, the performance of the proposed method is discussed. The position errors for the INS only, WSN, EKF, IEKF, and the proposed method are shown in Figure 5.

The east and north position errors of five estimation strategies in the first trajectory are shown in Figures 5(a) and 5(b), respectively. From these figures, it can be seen easily that the INS position error is accumulated. WSN is able to maintain the accuracy of position. It is evident that both the EKF and the IEKF are effective in reducing the position error compared with WSN. The errors for the proposed method are smaller than the ones for the IEKF. Figures 5(c) 


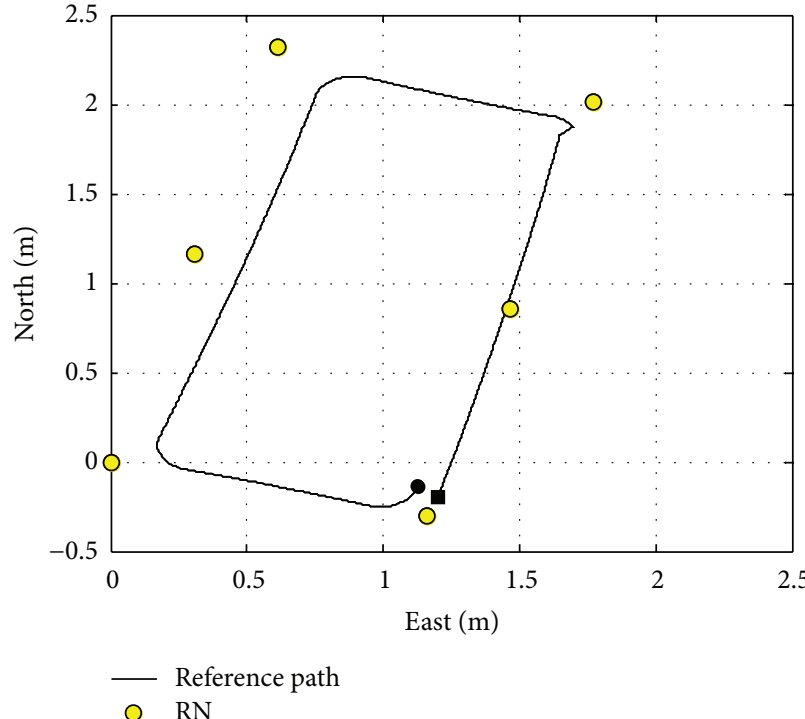

(a)

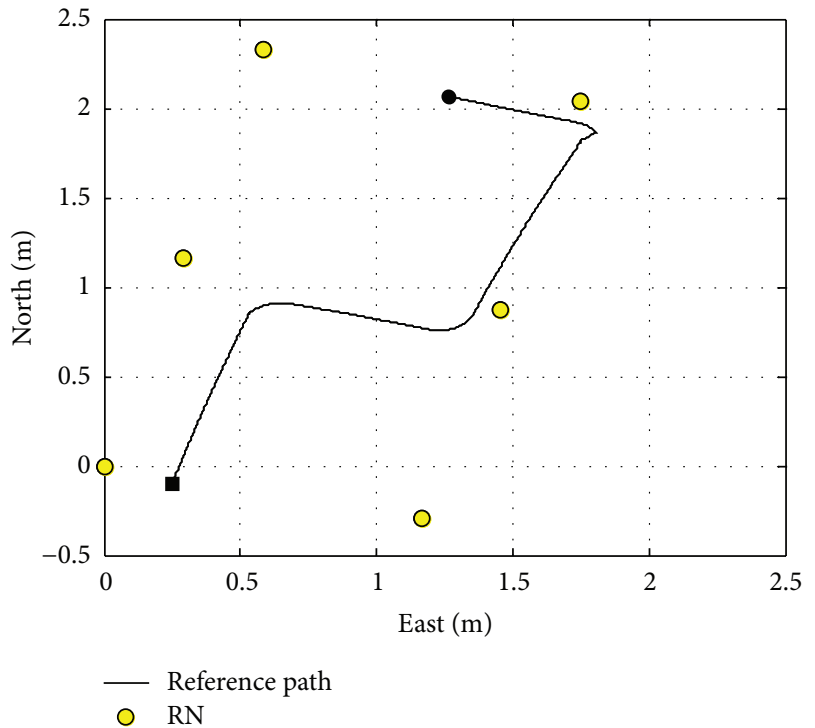

(b)

Figure 4: The trajectory of the real test.

TABLE 1: Comparison of five estimation strategies in terms of position error.

\begin{tabular}{|c|c|c|c|c|c|}
\hline \multirow{3}{*}{ Method } & \multicolumn{5}{|c|}{ RMSE (m) } \\
\hline & \multicolumn{2}{|c|}{ The first trajectory } & \multicolumn{2}{|c|}{ The second trajectory } & \multirow{2}{*}{ Mean } \\
\hline & East & North & East & North & \\
\hline INS only & 0.5912 & 0.4590 & 0.2108 & 0.3179 & 0.3947 \\
\hline WSN & 0.1132 & 0.0787 & 0.1065 & 0.0697 & 0.0920 \\
\hline EKF & 0.0721 & 0.0639 & 0.0736 & 0.0582 & 0.0670 \\
\hline IEKF & 0.0433 & 0.0433 & 0.0462 & 0.0360 & 0.0422 \\
\hline The proposed method & 0.0333 & 0.0290 & 0.0309 & 0.0249 & 0.0295 \\
\hline
\end{tabular}

TABLE 2: Comparison of five estimation strategies in terms of velocity error.

\begin{tabular}{|c|c|c|c|c|c|}
\hline \multirow{3}{*}{ Method } & \multicolumn{5}{|c|}{$\operatorname{RMSE}(\mathrm{m} / \mathrm{s})$} \\
\hline & \multicolumn{2}{|c|}{ The first trajectory } & \multicolumn{2}{|c|}{ The second trajectory } & \multirow{2}{*}{ Mean } \\
\hline & East & North & East & North & \\
\hline INS only & 0.1391 & 0.1682 & 0.1400 & 0.0957 & 0.1358 \\
\hline WSN & 0.0595 & 0.0854 & 0.0650 & 0.0794 & 0.0723 \\
\hline EKF & 0.0441 & 0.0539 & 0.0424 & 0.0437 & 0.0460 \\
\hline IEKF & 0.0425 & 0.0556 & 0.0412 & 0.0482 & 0.0469 \\
\hline The proposed method & 0.0445 & 0.0546 & 0.0420 & 0.0462 & 0.0468 \\
\hline
\end{tabular}

and 5(d) display the east and north position errors of five estimation strategies in the second trajectory. Similar to the first trajectory, it is evident that the proposed method has the smallest error.

The comparison of five estimation strategies in terms of position error is shown in Table 1. The results show that the proposed method has the lowest error in east and north direction respectively. The mean root-mean-square error (RMSE) of position for the proposed method is $0.0295 \mathrm{~m}$. It reduces the mean RMSE of position by about $92.53 \%, 67.93 \%$,
$55.97 \%$, and $30.09 \%$ compared with the INS only, WSN, EKF, and IEKF.

Table 2 shows the comparison of five estimation strategies in terms of velocity error. It can be seen that the EKF, IEKF, and the proposed method are able to reduce the velocity error compared with the IN S and the WSN, respectively. The result shows that the mean RMSE of velocity for the proposed method is $0.0468 \mathrm{~m} / \mathrm{s}$. However, the velocity estimation accuracy for the EKF, IEKF, and the proposed method is close. 


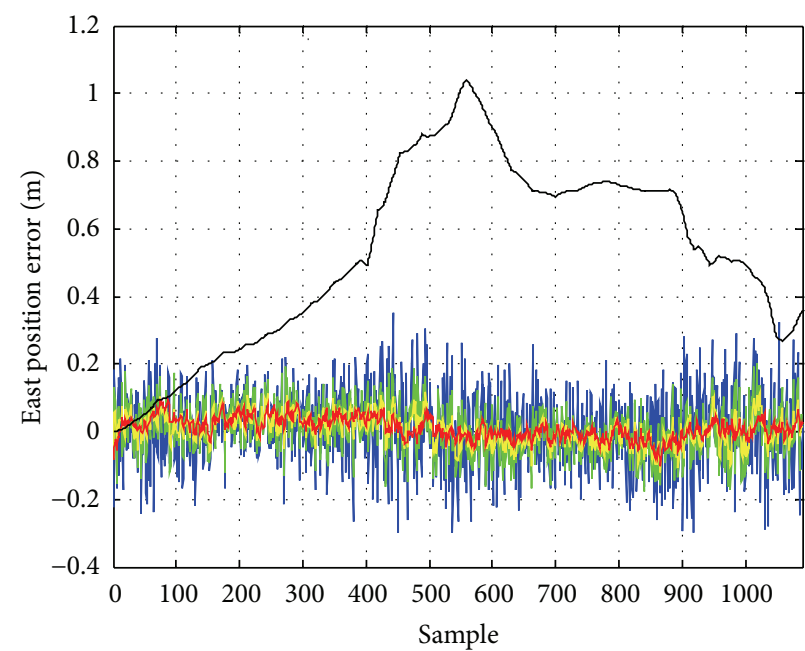

(a)

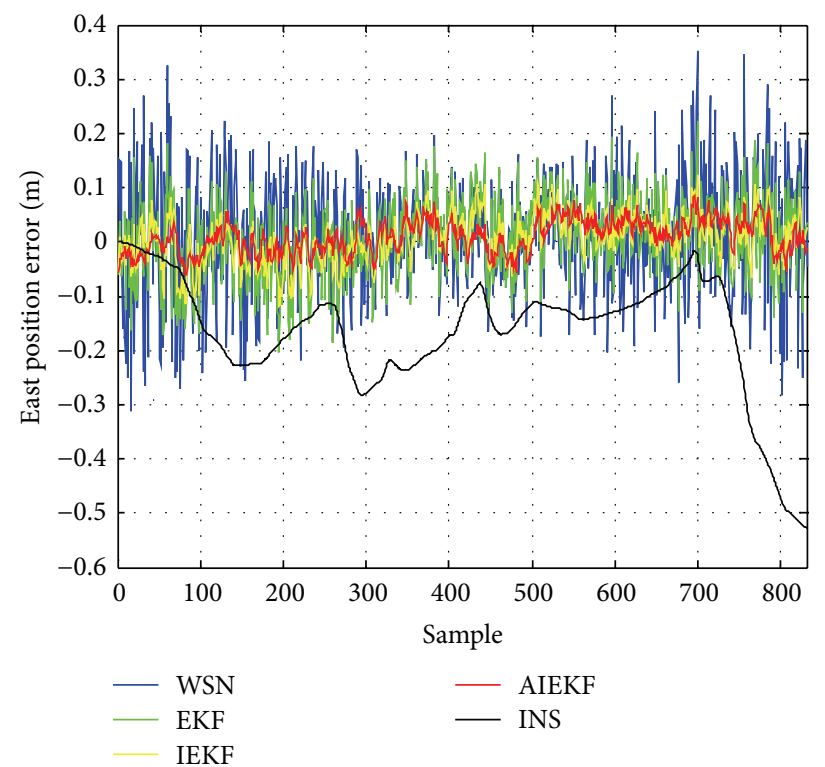

(c)

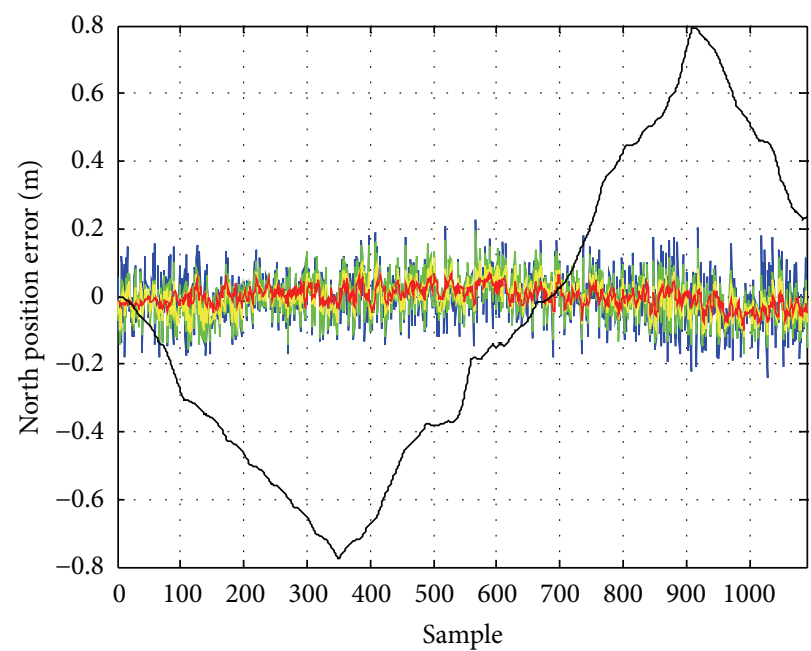

(b)

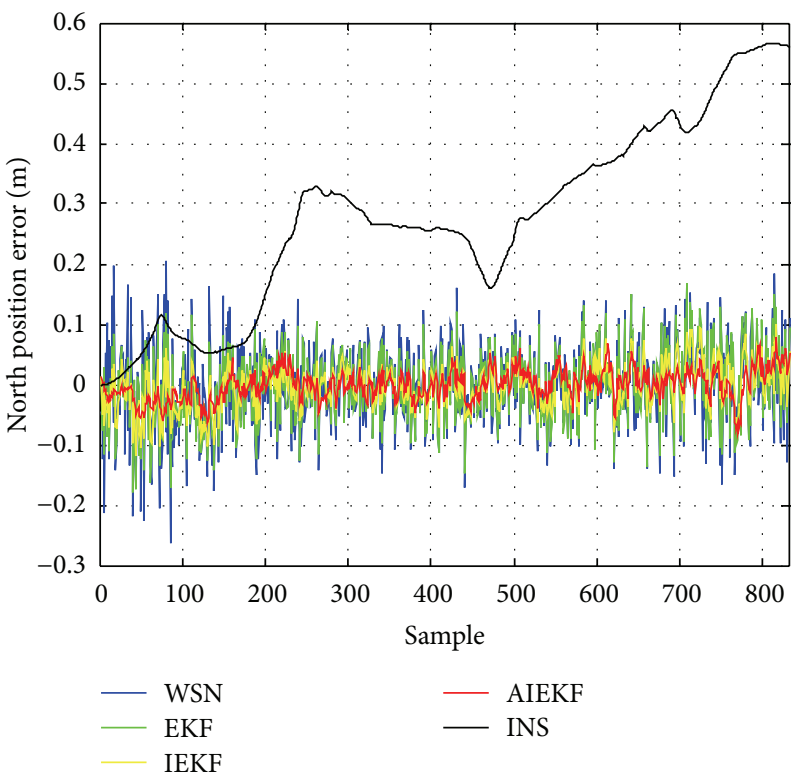

(d)

Figure 5: The position errors for the INS only, WSN, EKF, IEKF, and the proposed method. (a) and (b) The first trajectory. (c) and (d) The second trajectory.

\section{Conclusions}

In this work, the noise statistics estimator is employed into the IEKF to combine the advantages of the AEKF and the IEKF. Then, the AIEKF is used in INS/WSN integrated system. The experimental results show that the proposed method is effective in reducing the position error compared with the INS only, WSN, EKF, and IEKF; however, the velocity estimation accuracy for the EKF, IEKF, and the proposed method is close.

\section{Conflict of Interests}

The authors of the paper do not have a direct financial relation that might lead to a conflict of interests for any of the authors.

\section{Acknowledgments}

This work was supported in part by the National Natural Science Foundation of China (nos. 51375087, 41204025, and 50975049), Ocean Special Funds for Scientific Research on Public Causes (no. 201205035-09), Specialized Research Fund for the Doctoral Program of Higher Education (no. 20110092110039), the 52 and China Postdoctoral Science Foundation (no. 2012M520967), and the Program Sponsored for Scientific Innovation Research of College Graduate in Jiangsu Province, China (no. CXLX_0101).

\section{References}

[1] A. G. Quinchia, G. Falco, E. Falletti, F. Dovis, and C. Ferrer, "A comparison between different error modeling of MEMS applied 
to GPS/INS integrated systems," Sensors, vol. 13, no. 8, pp. 95499588, 2013.

[2] D.-J. Jwo, C.-F. Yang, C.-H. Chuang, and C. H. Lee, "Performance enhancement for ultra-tight GPS/INS integration using a fuzzy adaptive strong tracking unscented Kalman filter," Nonlinear Dynamics, vol. 73, no. 1-2, pp. 377-395, 2013.

[3] X. Chen, C. Shen, and Y. Zhao, "Study on GPS/INS system using novel filtering methods for vessel attitude determination," Mathematical Problems in Engineering, vol. 2013, Article ID 678943, 8 pages, 2013.

[4] D. J. Jwo, C. W. Hu, and C. H. Tseng, "Nonlinear filtering with IMM algorithm for ultra-tight GPS/INS integration: regular paper," International Journal of Advanced Robotic Systems, vol. 10, article 222, 2013.

[5] Y. Zhang, P. Agarwal, V. Bhatnaga, S. Balochian, and J. Yan, "Swarm intelligence and its applications," The Scientific World Journal, vol. 2013, Article ID 528069, 3 pages, 2013.

[6] C. M. Bishop, Neural Networks For Pattern Recognition, Oxford University Press, New York, NY, USA, 1995.

[7] S. Haykin, Neural Networks-A Comprehensive Foundation, IEEE Press, New York, NY, USA, 1994.

[8] A. Noureldin, A. El-Shafie, and M. Bayoumi, "GPS/INS integration utilizing dynamic neural networks for vehicular navigation," Information Fusion, vol. 12, no. 1, pp. 48-57, 2011.

[9] Z.-K. Xu, Y. Li, C. Rizos, and X. Xu, "Novel hybrid of LSSVM and kalman filter for GPS/INS integration," Journal of Navigation, vol. 63, no. 2, pp. 289-299, 2010.

[10] Y. Zhang and L. Wu, "Classification of fruits using computer vision and a multiclass support vector machine," Sensors, vol. 2012, no. 9, pp. 12489-12505, 2012.

[11] Y. Zhang, S. Wang, G. Ji, and Z. Dong, "An MR brain images classifier system via particle swarm optimization and kernel support vector machine," The Scientific World Journal, vol. 2013, Article ID 130134, 9 pages, 2013.

[12] S. J. Kim and B. K. Kim, "Accurate hybrid global self-localization algorithm for indoor mobile robots with two-dimensional isotropic ultrasonic receivers," IEEE Transactions on Instrumentation and Measurement, vol. 60, no. 10, pp. 3391-3404, 2011.

[13] A. R. J. Ruiz, F. S. Granja, J. C. P. Honorato, and J. I. G. Rosas, "Accurate pedestrian indoor navigation by tightly coupling foot-mounted IMU and RFID measurements," IEEE Transactions on Instrumentation and Measurement, vol. 61, no. 1, pp. 178-189, 2012.

[14] F. Chen and M. W. Dunnigan, "Comparative study of a slidingmode observer and Kalman filters for full state estimation in an induction machine," IEE Proceedings: Electric Power Applications, vol. 149, no. 1, pp. 53-64, 2002.

[15] Z. Chen, R. Rodrigo, V. Parsa, and J. Samarabandu, "Using ultrasonic and vision sensors within extended kalman filter for robot navigation," Canadian Acoustics, vol. 33, no. 3, pp. 28-29, 2005.

[16] H. Shao, D. Kim, and K. You, "TDOA/FDOA geolocation with adaptive extended Kalman filter," Communications in Computer and Information Science, vol. 121, pp. 226-235, 2010.

[17] A. Gelb, Applied Optimal Estimation, The MIT Press, Cambridge, Mass, USA, 1974.

[18] Y. Xu, X. Y. Chen, and Q. H. Li, "Unbiased tightly-coupled INS/WSN integrated navigation based on extended Kalman filter," Journal of Chinese Inertial Technology, vol. 20, no. 3, pp. 292-299, 2012. 

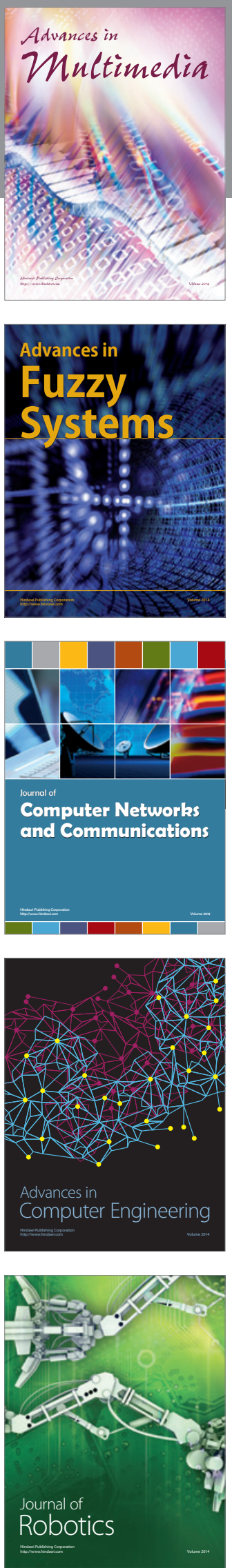

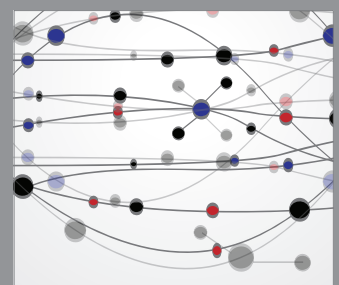

The Scientific World Journal
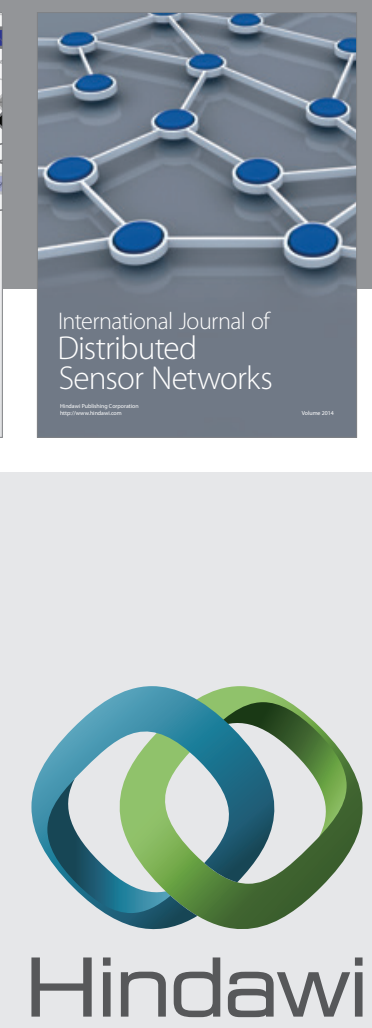

Submit your manuscripts at

http://www.hindawi.com
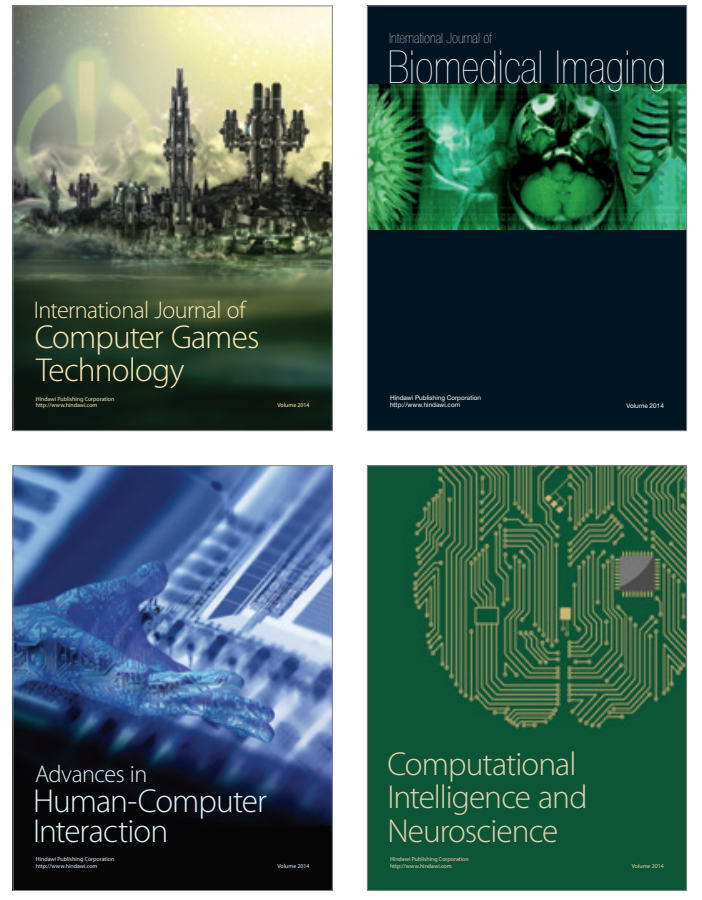
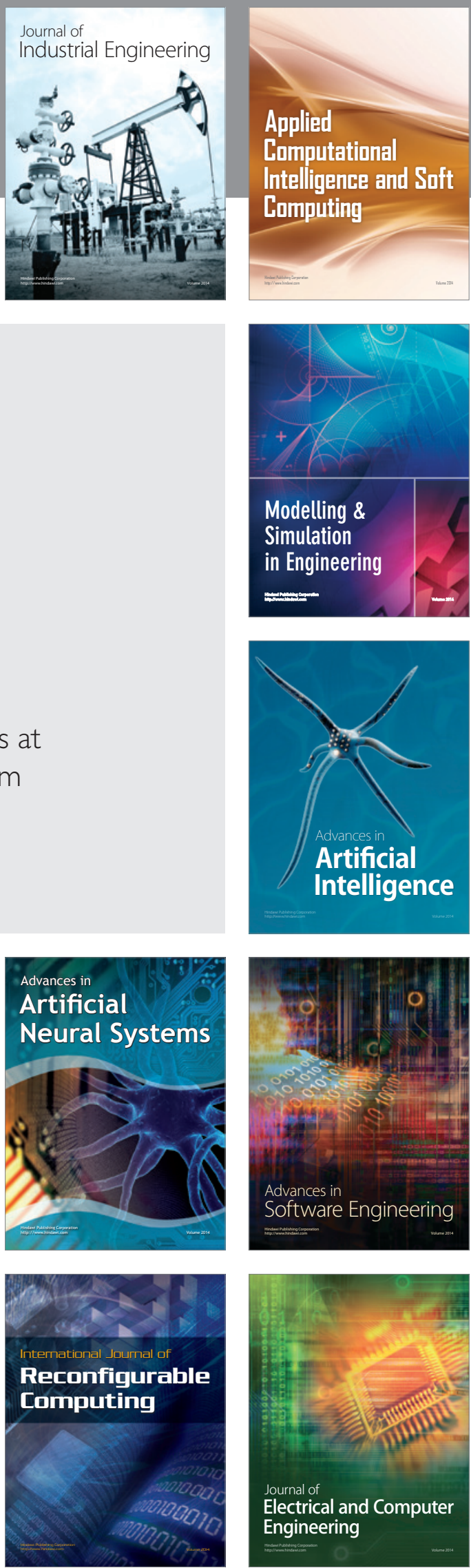